\title{
Treatment benefit or survival of the fittest: what drives the time-dependent decrease in serious infection rates under TNF inhibition and what does this imply for the individual patient?
}

\author{
A Strangfeld, ${ }^{1}$ M Eveslage, ${ }^{1}$ M Schneider, ${ }^{2}$ H J Bergerhausen, ${ }^{3}$ T Klopsch, ${ }^{4}$ A Zink, ${ }^{1,5}$ \\ $\mathrm{J}_{\text {Listing }}{ }^{1}$
}

-Additional data are published online only. To view the file please visit the journal online (http://ard.bmj.com)

1Programmbereich Epidemiologie, Deutsches Rheuma-Forschungszentrum Berlin, ein Leibniz Institut, Berlin, Germany

${ }^{2}$ Klinik fuer Endokrinologie, Diabetologie und

Rheumatologie, HeinrichHeine-Universitaet Duesseldorf, Duesseldorf, Germany ${ }^{3}$ Klinik fuer Rheumatologie, Wedau Kliniken, Klinikum Duisburg, Duisburg, Germany ${ }^{4}$ Rheumatologist in private practice, Neubrandenburg, Germany

${ }^{5}$ Klinik fuer Rheumatologie und Klinische Immunologie, Charité Universitaetsmedizin Berlin, Berlin, Germany

\section{Correspondence to}

Dr A Strangfeld,

Programmbereich

Epidemiologie, Deutsches

Rheuma-Forschungszentrum Berlin, ein Leibniz Institut,

Charitéplatz 1,

10117 Berlin, Germany; strangfeld@drfz.de

Accepted 13 June 2011 Published Online First 25 July 2011
ABSTRACT

Objective To examine the risk of serious infection conveyed by tumour necrosis factor $\alpha$ (TNF $\alpha$ ) inhibitors in the treatment of rheumatoid arthritis (RA).

Methods Data from patients with RA enrolled in the German biologics register RABBIT were used for analysis. Baseline patient characteristics, time-varying risk factors (treatment changes, functional capacity) and selection processes caused by dropout, death or switching to nonanti-TNF treatment were taken into account to estimate the adjusted incidence rate ratios (IRR $\left.\mathrm{adj}_{\mathrm{j}}\right)$ of serious infection during treatment with TNF inhibitors compared with non-biological disease-modifying antirheumatic drug treatment.

Results Data were available on 5044 patients, in whom 392 serious infections occurred. The crude rates of serious infections in patients treated with TNF inhibitors declined over the first 3 years of observation (from 4.8 to $2.2 / 100$ patient-years). This decline was driven by (1) treatment termination or loss to follow-up in patients at increased risk and (2) a risk reduction through decreasing glucocorticoid doses and improvement in function. Adjusted for selection processes and time-varying risk factors, the following parameters assessed at baseline (age, chronic diseases) or at follow-up prior to the infection were significantly associated with an increased risk: age $>60$ years, chronic lung or renal disease, low functional capacity, history of serious infections, treatment with glucocorticoids (7.5-14 mg/day, IRR ${ }_{\text {adj }} 2.1$ (95\% Cl 1.4 to 3.2$) ; \geq 15 \mathrm{mg} /$ day, IRR $\operatorname{IRdj}_{\text {ad }} 4.7$ (95\% Cl 2.4 to 9.4)) and treatment with TNF $\alpha$ inhibitors (IRR ${ }_{\text {adj }} 1.8(95 \%$ Cl 1.2 to 2.7)).

Conclusion Reasons for the decline in infection rates observed at the group level were identified. The results enable expected infection rates to be calculated in individual patients based on their risk profiles.

\section{INTRODUCTION}

A decade ago tumour necrosis factor (TNF) inhibitors were approved for the treatment of rheumatoid arthritis (RA). Uncertainties regarding the specific risks of these new agents led to intensified efforts to investigate their safety. Meta-analyses of randomised controlled trials (RCTs) were undertaken, and various data sources including claims databases were used to assess the risk of serious adverse events (eg, serious infections) possibly associated with these agents. ${ }^{1-11}$ One major innovation was the establishment of biologics registers to evaluate the safety and effectiveness of these drugs under the conditions of daily rheumatological care. ${ }^{12}$ Upon analysis of these data, it became increasingly clear that the question of how a drug affects the risk of a specific adverse event is far more complex than evaluating the efficacy of the drug. Some of the methodological difficulties we faced while analysing real-world data will be described in this paper using the example of examining how antiTNF therapy affects the risk of serious infection.

An increased rate of serious infections with antiTNF therapy compared with conventional diseasemodifying antirheumatic drug (DMARD) therapy was found in a meta-analysis of RCTs, ${ }^{1}$ in an analysis of claims data ${ }^{8}$ and in observational data. ${ }^{3} 69$ Other findings were in contrast to these results 21011 or reported a decline in the infection risk over time in patients treated with TNF inhibitors, 5813 and therefore raised the question whether or not the risk is increased only during the first months of treatment. 5713

The first aim of our study therefore was to determine whether or not there is a methodological or clinical explanation for this decline in risk and, if so, what the relative contributions might be.

Our second question was how the risk reduction seen in the cohorts is reflected at the level of the individual patient. Patient demographics, clinical features and follow-up information such as treatment response and patient use of additional medications were studied to calculate the expected incidence rates of serious infections for defined subgroups of patients. The aim of this approach was to enable the treating physician to assess the magnitude of infection risk that he or she imposes on a patient when making specific treatment decisions.

\section{METHODS \\ Patients}

The analysis was based on patients with RA enrolled in the German biologics register RABBIT, an ongoing prospective cohort study, at the start of treatment with a biological agent or a conventional DMARD, between 1 May 2001 and 31 December 2006. Patients were followed up independent of any change in their treatment regimes. Observation time following a start of treatment with a non-antiTNF biological agent was excluded. Patients treated with anakinra at baseline $(n=89)$ and those for whom only baseline data were available $(n=141)$ were excluded. 


\section{Assessments}

At baseline and at predefined points of follow-up (at 3, 6 months and thereafter every 6 months), rheumatologists assessed the clinical status of the patient including the components of the disease activity score based on 28-joint counts (DAS28), ${ }^{14}$ reported treatment details and adverse events. Patients assessed, among other items, their functional capacity as a percentage of full function by means of the Hannover Functional Status Questionnaire (Funktionsfragebogen Hannover (FFbH)). ${ }^{15}{ }^{16}$ Reasons for dropout from the study and causes of death were ascertained by contacting health authorities. Follow-up data (including serious infections) reported prior to 1 November 2009 were included. An infection was attributed to anti-TNF treatment when the treatment was either ongoing or terminated $\leq 3$ months prior to infection. A patient who switched from anti-TNF treatment to treatment with non-biological DMARDs contributed to anti-TNF exposure time until 3 months after switching and to DMARD exposure time thereafter. A DMARD-treated patient who switched to antiTNF treatment also contributed time to both exposure groups (for further details see Strangfeld et a 17 or Listing et $a^{\beta}$ ).

\section{Statistical analysis}

Three different statistical models developed in a complementary fashion were used. In model A we adjusted the data only for differences between the anti-TNF and DMARD groups at the start of treatment (confounding by indication). A propensity score, as described earlier, ${ }^{18}$ was used for adjustment. Further adjustment was made for risk factors for infection (table 3). In model $\mathrm{B}$ we also considered changes in glucocorticoid dosage and changes in functional status during follow-up as timevarying covariables. The differences between models $A$ and $B$ in the estimates of the trend parameter were used to calculate the relative contribution of clinical improvement to the decreased risk of infection. In the fully adjusted model $\mathrm{C}$, generalised estimation equations (GEEs) were applied to a weighted sample of patients. In addition to model $\mathrm{B}$, this model takes into account confounding by anti-TNF treatment termination, the impact of dropout and previous serious infections. ${ }^{19}$ We defined 'dropout' as either loss to follow-up, death or switching to non-anti-TNF therapy.

For each patient and each 6-month follow-up period we calculated the probability of being in a certain status. Using logistic regression we estimated the probabilities of being a dropout with and without accounting for gender and patient characteristics at follow-up (occurrence of serious infections, DAS28, functional status). We calculated weights as ratios of both probabilities. The weights for different time periods were combined as described in detail by Molenberghs et al. ${ }^{20}$ Again, for each 6-month period

Table 1 Baseline characteristics of patients

\begin{tabular}{|c|c|c|c|c|c|c|c|}
\hline & \multicolumn{4}{|c|}{ Unweighted sample } & \multicolumn{3}{|l|}{ Weighted sample } \\
\hline & Anti-TNF & DMARD & Total & p Value & Anti-TNF & DMARD & p Value \\
\hline $\mathrm{N}$ & 3271 & 1773 & 5044 & & 3271 & 1773 & \\
\hline Weights, mean (SD) & $1(0)$ & $1(0)$ & $1(0)$ & - & $0.99(0.8)$ & $1.0(1.2)$ & 0.15 \\
\hline Female, n (\%) & $2556(78.1)$ & $1394(78.6)$ & $3950(78.3)$ & 0.69 & $2612.2(78.8)$ & $1519.4(77.6)$ & 0.29 \\
\hline Age & $53.8(12.3)$ & $56.2(11.5)$ & $54.6(12.1)$ & $<0.0001$ & $54.4(12.3)$ & $54.6(12.1)$ & 0.47 \\
\hline Median (IOR) disease duration, years & $9(5,16)$ & $6(3,12)$ & $8(4,15)$ & $<0.0001$ & $8(4,15)$ & $8(4,16)$ & 0.45 \\
\hline Median (IOR) follow-up, years & $3.1(2.1,4.9)$ & $3.3(2.5,5.0)$ & $3.1(2.4,5.0)$ & $<0.0001$ & $3(2.2,4.6)$ & $3.5(2.5,5)$ & $<0.0001$ \\
\hline Rheumatoid factor positive, $\mathrm{n}(\%)$ & $2624(80.2)$ & $1271(71.7)$ & $3895(77.2)$ & $<0.0001$ & $2620.4(79.0)$ & $1479.6(75.5)$ & 0.003 \\
\hline DAS28 & $5.7(1.2)$ & $5.1(1.3)$ & $5.5(1.3)$ & $<0.0001$ & $5.5(1.3)$ & $5.6(1.3)$ & 0.20 \\
\hline $\mathrm{FFbH}$ & $57.0(23.0)$ & $66.6(21.5)$ & $60.4(22.9)$ & $<0.0001$ & $60.0(22.8)$ & $58.7(24.3)$ & 0.05 \\
\hline Smoking ever, n (\%) & $1027(47.0)$ & $585(45.6)$ & $1612(46.4)$ & 0.43 & $1036.1(46.3)$ & $621.1(44.7)$ & 0.36 \\
\hline No of previous DMARDs & $3.3(1.3)$ & $1.8(1.0)$ & $2.8(1.4)$ & $<0.0001$ & $2.8(1.4)$ & $2.9(1.5)$ & 0.01 \\
\hline No of previous biologics & $0.23(0.6)$ & $0.01(0.1)$ & $0.14(0.4)$ & $<0.0001$ & $0.17(0.5)$ & $0.10(0.4)$ & $<0.0001$ \\
\hline Glucocorticoids 7.5-14 mg/day, n (\%) & $1027(31.4)$ & $386(21.8)$ & $1413(28.0)$ & $<0.0001$ & $938.6(30.0)$ & $404.1(25.4)$ & $<0.0001$ \\
\hline Glucocorticoids $\geq 15 \mathrm{mg} /$ day, $\mathrm{n}(\%)$ & $491(15.0)$ & $147(8.3)$ & $683(12.7)$ & $<0.0001$ & $465.5(14.9)$ & $146.6(9.2)$ & $<0.0001$ \\
\hline COPD, n (\%) & $162(5.0)$ & $87(4.9)$ & $249(4.9)$ & 0.94 & $168.9(5.1)$ & $86.8(4.4)$ & 0.28 \\
\hline Chronic lung diseases total, $\mathrm{n}(\%)$ & $246(7.5)$ & $112(6.3)$ & $358(7.1)$ & 0.11 & $247.7(7.5)$ & $135.9(6.9)$ & 0.47 \\
\hline Chronic renal disease, $\mathrm{n}(\%)$ & $139(4.3)$ & $31(1.8)$ & $170(3.4)$ & $<0.0001$ & $156.1(4.7)$ & $56.8(2.9)$ & 0.001 \\
\hline
\end{tabular}

Values are mean (SD) unless otherwise specified

The weights estimated for the first time period (0-6 months) were used to calculate the weighted sample (columns 6 and 7).

COPD, chronic obstructive pulmonary disease; DAS28, disease activity score based on 28 joint counts; DMARD, disease-modifying antirheumatic drug; FFbH, Hannover Functional Status Questionnaire measuring functional capacity as percentage of full function; TNF, tumour necrosis factor.

Table 2 Crude rates of serious infections per 100 patient-years (pyrs)

\begin{tabular}{|c|c|c|c|c|c|}
\hline & \multirow[b]{2}{*}{ Exposure time (pyrs) } & \multicolumn{3}{|c|}{ Serious infections } & \multirow{2}{*}{$\begin{array}{l}\text { Incidence rate } \\
\text { ratio (IRR) }\end{array}$} \\
\hline & & $\mathrm{n}$ & Per 100 pyrs & $95 \% \mathrm{CI}$ & \\
\hline \multicolumn{6}{|l|}{ Year 1} \\
\hline DMARD treatment & 1765 & 40 & 2.3 & 1.6 to 3.1 & 2.13 \\
\hline Anti-TNF agents & 3041 & 147 & 4.8 & 4.1 to 5.7 & \\
\hline \multicolumn{6}{|l|}{ Year 2} \\
\hline DMARD treatment & 1696 & 40 & 2.4 & 1.7 to 3.2 & 1.36 \\
\hline Anti-TNF agents & 2564 & 82 & 3.2 & 2.9 to 4.0 & \\
\hline \multicolumn{6}{|l|}{ Year 3} \\
\hline DMARD treatment & 1397 & 35 & 2.5 & 1.8 to 3.5 & 0.88 \\
\hline Anti-TNF agents & 2186 & 48 & 2.2 & 1.6 to 2.9 & \\
\hline
\end{tabular}

DMARD, disease-modifying antirheumatic drug; TNF, tumour necrosis factor. 


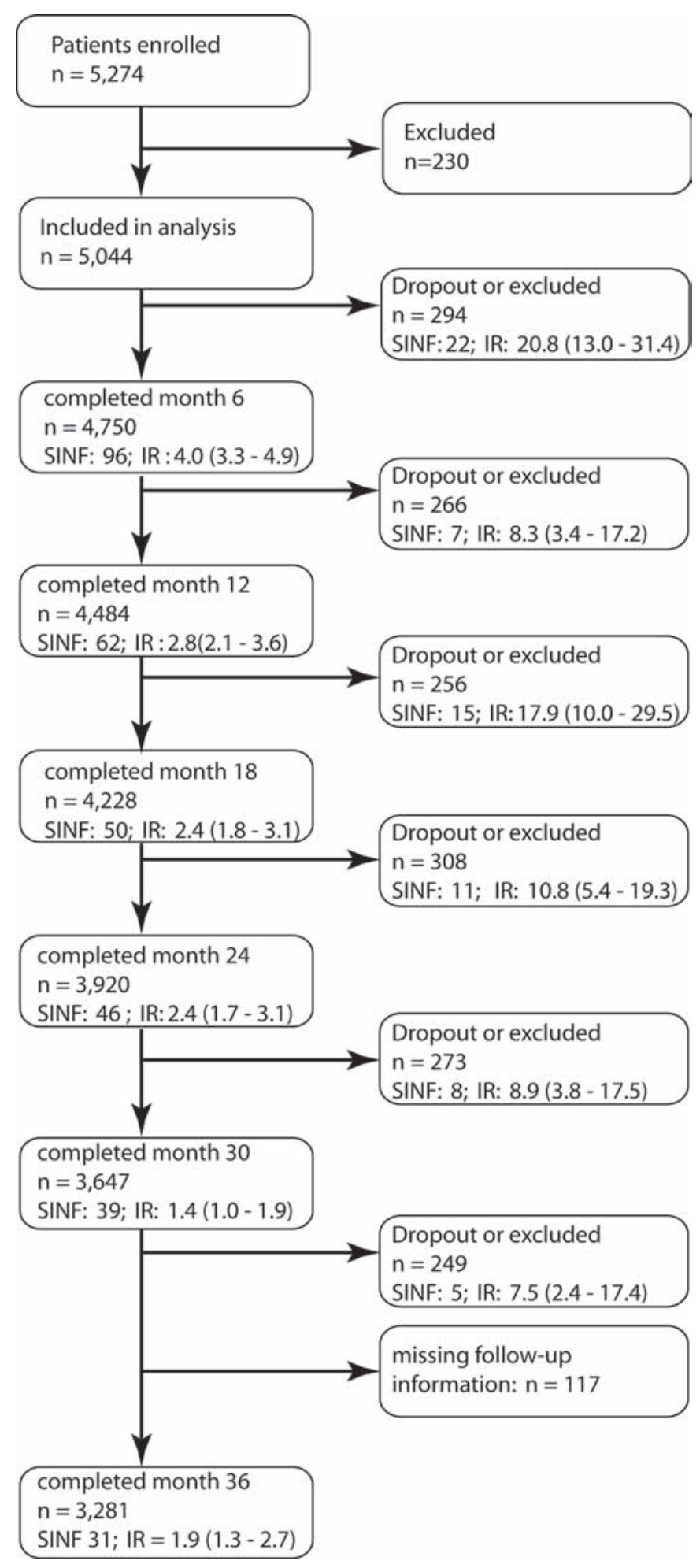

Figure 1 Flow chart of patients who dropped out of observation with rates of serious infections in patients who continued and those who dropped out. Dropouts include patients who switched to nonanti-tumour necrosis factor biological agents. IR, incidence rate; SINF, number of serious infections.

the likelihood of being treated with anti-TNF agents was calculated by taking and not taking into account covariables (age, number of DMARD failures and the patient characteristics listed above). This resulted in weights which account for treatment decisions. ${ }^{19} 20$ Both types of weights (for dropout and treatment groups) were uncorrelated $(|r|<0.1)$ and were then combined by multiplication to a final weight. By using this inverse probability weighting we obtained balanced samples of patients treated with or without TNF $\alpha$ inhibitors and of patients who continued or discontinued follow-up.

The application of multivariate GEE models requires a sufficient number of observed serious infections. We aimed to detect a $\geq 1.5$-fold increase in infection risk caused by TNF inhibitors or a trend in risk of similar size $(\leq 0.67=1 / 1.5)$ that would be comparable to other observations. ${ }^{2}$ The number of serious infections observed in the first and second years would therefore ensure sufficient power ( $80 \%$ ) for this analysis, whereas the total number of infections observed in the third year $(n=83)$ was too low to achieve sufficient power. Because multiple infections in individual patients are not independent of each other, the GEE models require an estimate of intrapatient correlations. The number of these correlations increased considerably when the data were analysed for all 3 years. Therefore, a robust estimate of the 3 -year infection risk was not possible. For both reasons (the small number of events and the high number of intracorrelations), we restricted the multivariate analysis to the first 2 years.

Owing to strict monitoring, information about DMARD or anti-TNF treatment exposure was complete in $>99 \%$ of all patients. For all follow-up time points within the first 2 years (in dropouts before their last visit), $5.6 \%$ of DAS28 scores, $3.7 \%$ of $\mathrm{FFbH}$ scores and $3.6 \%$ of glucocorticoid dosages were missing. These values were replaced using statistical imputation methods based on the expectation maximation algorithm..$^{20}$ SAS software version 9.2 (PROC GLIMMIX, PROC MI) was used for the computations. $p$ Values $<0.05$ were considered to be statistically significant.

\section{RESULTS}

\section{Patient characteristics and treatment status at study entry}

Between 1 May 2001 and 31 December 2006, 5274 patients were enrolled in RABBIT of whom 5044 met the inclusion criteria for this study. The average follow-up time was 2.6 years. Upon enrolment, patients treated with TNF $\alpha$ inhibitors differed significantly from those receiving conventional DMARDs with regard to age, disease duration, disease activity (DAS28), functional capacity $(\mathrm{FFbH})$ or number of failed previous DMARD treatments (table 1, columns $2-5$ ). The proportion of patients receiving higher doses of glucocorticoids was also significantly different. Inverse probability weighting was used for the final analysis and allowed for balanced anti-TNF and DMARD samples (table 1, columns 6-8).

\section{Trends in crude infection rates}

The crude rates of serious infections decreased significantly from the first to the second and third years in patients treated with TNF inhibitors, whereas these rates remained stable in the cohort of DMARD-treated patients (table 2). The decrease in the absolute rates of infection in patients treated with TNF inhibitors led to a decrease in the relative rates or incidence rate ratios (IRRs).

\section{Changes in patient risk profiles over time}

Overall, 1893 patients dropped out because of loss to follow-up, death or changing to non-anti-TNF biological agents within 3 years (figure 1). Dropouts experienced a significantly greater number of serious infections during the 6 months prior to dropping out than the patients who remained in the study. This association remained significant after adjustment for other risk factors of dropping out (male gender, elevated DAS28 scores, poor functional capacity) and repeated significance testing. The 

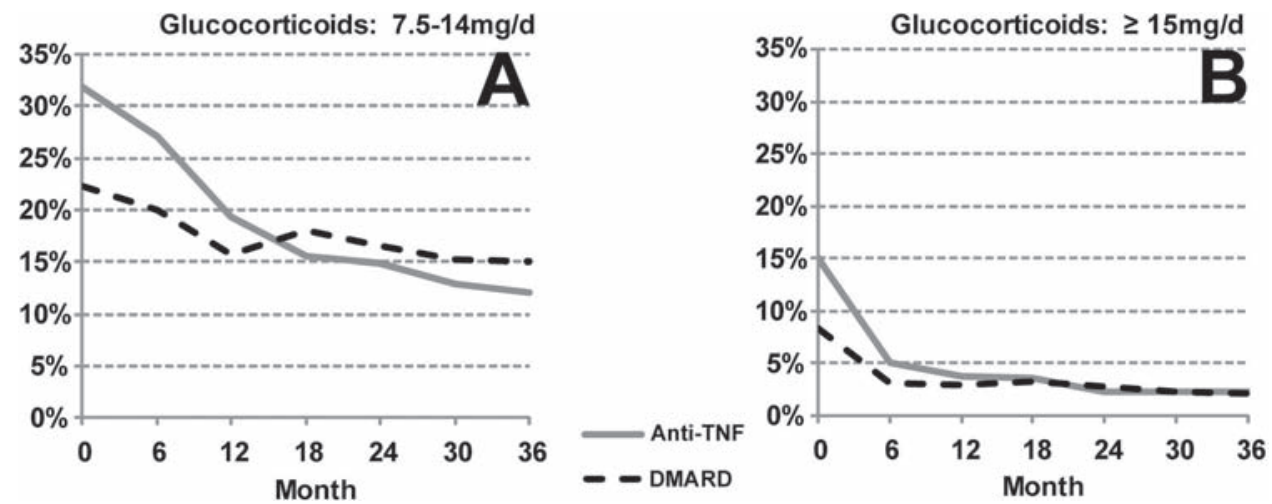

Figure 2 Decline in co-medication with glucocorticoids in patients who received a dose of (A) 7.5-14 mg/day or (\&) ! $15 \mathrm{mg} / \mathrm{day}$. DMARD, diseasemodifying antirheumatic drug; TNF, tumour necrosis factor.

Table 3 Adjusted incidence rate ratios (IRR) of developing a serious infection in the first or second year

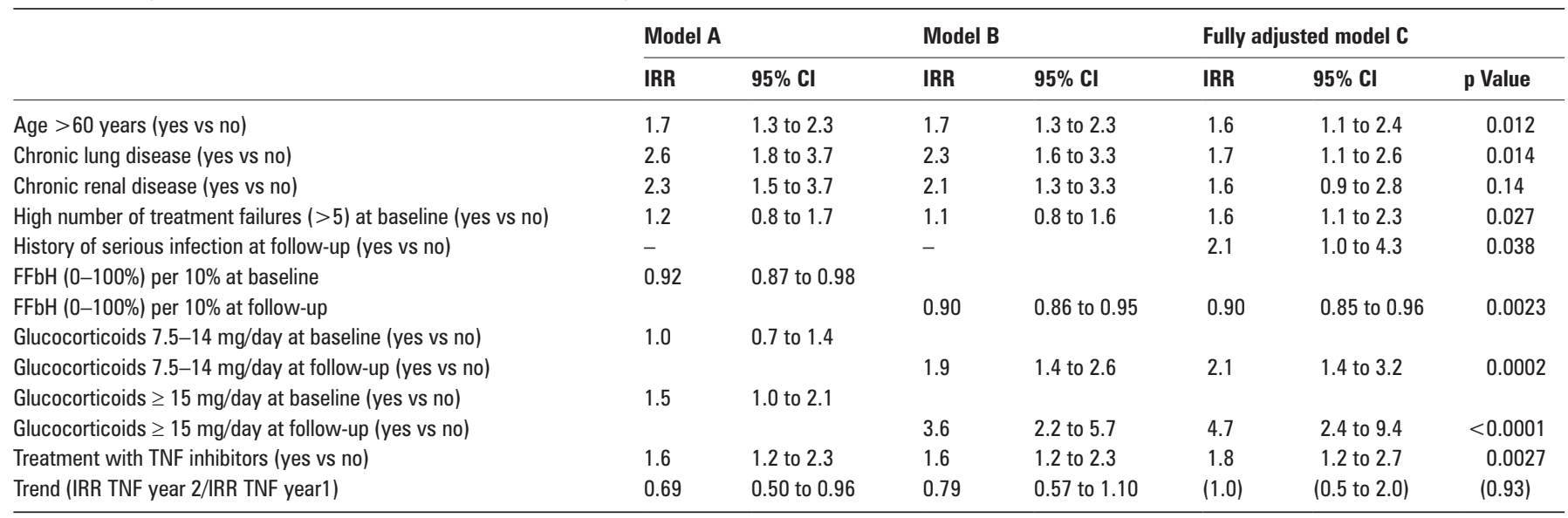

\footnotetext{
Model A: only baseline characteristics were used for calculation and adjustment.

Model B: time-dependent use of glucocorticoids and FFbH were considered in the model. No adjustment for DMARD/anti-TNF treatment adaptations and dropout processes at follow-up.

Model C: fully adjusted model. Adjustment for time-varying risk factors as in model B and for treatment adaptations and dropout processes (see Methods section for further details). DMARD, disease-modifying antirheumatic drug; FFbH, Hannover Functional Status Questionnaire measuring functional capacity as percentage of full function; TNF, tumour necrosis factor.
}

adjusted $\mathrm{OR}$ for dropping out after a serious infection calculated for each 6-month period ranged from 2.7 (95\% CI 1.2 to 6.2 ) to 4.7 (95\% CI 2.5 to 8.8$)$.

Furthermore, patients who developed serious infections were more likely to switch from anti-TNF treatment to DMARD treatment. Since patients with prior serious infection were at increased risk of developing a further serious infection (see below), this switching led to changes in the risk profiles of the treatment groups (data not shown).

Another factor leading to a change in patient risk profile was the decreasing number of patients who received higher doses of glucocorticoids. The decline in the percentage of patients who needed this additional treatment was far more pronounced in the anti-TNF group (figure 2).

\section{Multivariate analysis}

To estimate the relative contributions of changes in the clinical status of the patients and of selection processes to the timevarying risk of infection, we applied three different statistical models (see Methods section). Based on model A which adjusted only for the baseline characteristics of the patients, we observed a statistically significant $31 \%$ decrease in the IRRs from year 1 to year 2 in the anti-TNF treatment group compared with the DMARD group (table 3). This decrease was comparable to the decrease in crude IRRs (table 2). However, model A disregards the considerable changes in glucocorticoid dose during the follow-up period (figure 2) and therefore misclassifies exposure at follow-up. Taking into account the time-varying change of glucocorticoid dose and the improvement in functional capacity (model B), approximately one-third $(1-(1-0.79) /(1-0.69))$ of the decrease observed in model A can be attributed to the efficacy of TNF inhibitors. Dropout and treatment changes (either to non-anti-TNF biological agents or from anti-TNF to conventional DMARD treatment) in patients at risk of infection are responsible for the remaining two-thirds of the decline.

The additional adjustment for dropout processes and treatment adaptations in model $\mathrm{C}$ led to changes in the IRRs compared with model $\mathrm{B}$ which were observed in the expected direction (table 3). Overall, these changes resulted in an IRR for trend of 1.0, suggesting that there is no time-dependent decline in the risk of infection with TNF inhibition if all risk factors are controlled for (table 3).

We found a significantly increased risk of serious infection in patients who had already developed a serious infection earlier in the observation period. Furthermore, older age, chronic lung disease, chronic renal disease and a high number of previous treatment failures increased the risk, whereas a good functional status reduced the risk. The risk was significantly increased in 


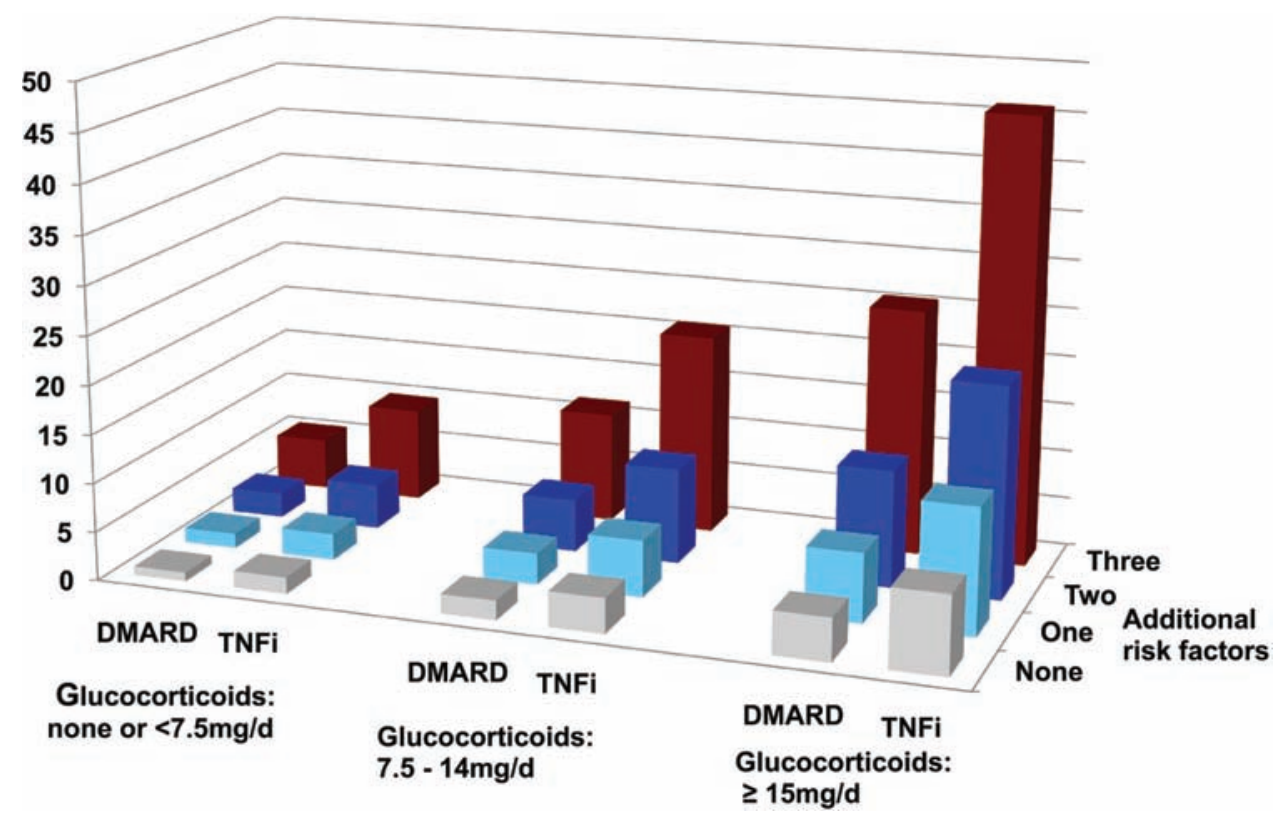

Figure 3 Estimated incidences of serious infections in 100 patients per year by treatment and risk profile. Additional risk factors are one or two of the following: age >60 years, chronic lung disease, chronic renal disease or high number of treatment failures; three risk factors: two of the above risk factors plus prior serious infections. DMARD, disease-modifying antirheumatic drug; TNFi, tumour necrosis factor inhibitor.

patients treated with glucocorticoids in a dose of $\geq 15 \mathrm{mg} /$ day (IRR=4.7) or 7.5-14 $\mathrm{mg} /$ day $(\mathrm{IRR}=2.1)$ and in patients treated with TNF inhibitors (IRR=1.8). No significant increase in risk was observed for treatment with lower doses of glucocorticoids $(\operatorname{IRR}=1.1(0.8 ; 1.7))$ or in patients with other co-morbid conditions (eg, diabetes, $\mathrm{IRR}=1.3(0.8 ; 2.0))$. Higher disease activity was not directly associated with an increased infection risk but indirectly via the use of glucocorticoids and decline in function.

\section{Absolute risks in different groups of patients}

The fully adjusted model $\mathrm{C}$ allows estimation of relative risks (IRRs) and also the calculation of expected incidence rates of serious infection for individual patients depending on their risk profiles. As the IRRs of model C are not time-dependent, these incidence rates are also not time-dependent. They only change in a patient when the risk factors she or he is exposed to change.

The rates shown in figure 3 (and in file 1 in the online supplement) reflect how the infection risk in an individual patient is influenced by treatment with different doses of glucocorticoids or by treatment with anti-TNF agents compared with DMARDs. The rates further describe how the risk increases if a patient has one, two or three risk factors for serious infection (greater age, comorbid conditions such as chronic lung disease or chronic renal disease, or history of serious infection). The risk of infection increases steadily with the number of risk factors and with the dose of glucocorticoids, both in the anti-TNF and DMARD treatment groups.

The number of patients with two or more risk factors and treatment with glucocorticoids in a dose of $\geq 15 \mathrm{mg} /$ day was rather low (70 exposed to anti-TNF, 39 treated with DMARDs). Nevertheless, the estimated incidence rates from model C were in accordance with the observed incidence rates in these patients. Taking into account the duration of exposure to high-dose glucocorticoids, the specific risk factors and the functional capacity of these patients, we would have expected 12.8 (95\% CI 6.0 to 27.6) serious infections in patients exposed to anti-TNF agents. This modelling aligned well with the 13 serious infections that were actually observed in these 70 patients. A similar result was found for the 39 DMARD-treated patients in whom three serious infections were observed and 3.7 (95\% CI 1.6 to 8.5 ) were predicted by the model.

\section{DISCUSSION}

Long-term safety data from observational studies reflect timedependent changes in the risk profiles of individual patients as well as changes in the composition of the cohorts. Our study shows how variable the results from these studies can be, depending on the statistical model applied to adjust for differences between groups.

Using our statistical model A, we saw a similar decline in the absolute and relative risk of infection in the anti-TNF group in the second year of treatment, as has been reported from other observational data. ${ }^{513}$ Taking into account changes in clinical status (model B) and the effect of treatment changes and dropouts (model C), we were able to explain this decrease and to estimate the relative contributions of both processes. We found that approximately one-third of the decrease in risk was caused by improvement in the clinical status and reduction of concomitant glucocorticoid therapy (mirroring a reduction of risk in individual patients). The remaining two-thirds of the decrease in risk could be explained by selective switching of patients who were at increased risk of infection. Both processes (clinical improvement and depletion of susceptible patients) led to a 'healthy drug survivor effect' in the anti-TNF cohort-that is, those patients who did well and responded to treatment remained under therapy. Of note, such a 'healthy drug survivor effect' is also present in extension studies of RCTs and might explain the contrasting results of two meta-analyses. ${ }^{12}$ With regard to the question as to what are the risk factors of serious infection, we found that age, functional status and comorbidity contributed significantly to the overall infection risk, in addition to treatment with TNF inhibitors or glucocorticoids.

The adjusted relative risk of treatment with TNF inhibitors compared with that of conventional DMARDs was 1.8. 
Furthermore, the adjusted relative risk of glucocorticoids was 2.1 for a dose of $\geq 7.5 \mathrm{mg} /$ day and 4.7 for a dose of $\geq 15 \mathrm{mg}$ /day. This result underlines the high risk conveyed by glucocorticoids that has also been addressed by other researchers. ${ }^{8-11} 2122$

The risk imposed on a patient by one treatment must be balanced against the risk conferred by an alternative treatment. For example, consider a patient with highly active RA, aged 65, treated with methotrexate and $10 \mathrm{mg} /$ day glucocorticoids. This patient has an 'average' risk of developing 0.032 serious infections per year. This risk increases to 0.058 if anti-TNF treatment is started, but it may decrease to 0.027 if glucocorticoid treatment can be reduced below $7.5 \mathrm{mg} /$ day due to the response to anti-TNF treatment. The number needed to harm in this example decreases from 1 out of 31 with DMARD treatment plus $10 \mathrm{mg}$ glucocorticoids to 1 out of 17 after start of anti-TNF and increases again to 1 out of 37 after stopping glucocorticoid treatment and maintaining TNF inhibition.

According to our data, improvement in function as a result of effective treatment can reduce the risk of serious infection significantly and to a greater extent than improvement in disease activity measured by the DAS28. This result is clinically plausible when one considers that immobility is a strong risk factor for developing comorbidities such as pneumonia or urogenital infections. Cardiovascular risk factors, as discussed prepviously, ${ }^{710}$ are linked to age and were therefore not identified in our data.

Our analysis has some limitations. We investigated only the overall infection risk and were therefore not able to consider site-specific risk factors. There was also limited statistical power to analyse the data from the third year. We did not investigate the three TNF inhibitors separately. Although different effects on the reactivation of tuberculosis ${ }^{23}$ and viral infections ${ }^{17}$ have been reported for adalimumab, infliximab and etanercept, the overall infection risk appears to be similar. ${ }^{3} 513$

The important message from this study to the practising physician is that the time-dependent decline in risk seen in cohorts of patients treated with anti-TNF agents is partially caused by changes in the case mix of these cohorts and cannot directly be transferred to individual patients. Independent of treatment duration, TNF inhibition imposes an increased risk of serious infection on a patient which must be balanced against other risks resulting from co-medication, in particular higher doses of glucocorticoids and active disease.

Our data provide, for the first time, detailed information about the expected absolute risk of serious infection in subgroups of patients. This work therefore helps rheumatologists to identify patients at increased risk and to avoid combinations of treatments that confer a very high risk in predisposed patients.

Acknowledgements The authors acknowledge the invaluable contributions of all
participating consultant rheumatologists. In particular, they would like to thank those
who enrolled 25 patients or more: Ulrich von Hinüber, Hildesheim; Andreas Krause,
Immanuel Hospital Berlin; Karin Babinsky, Halle; Arnold Bussmann, Geilenkirchen;
Hans Peter Tony, Medizinische Poliklinik der Universität Würzburg; Katja Richter,
Universitätsklinikum Carl Gustav Carus, Dresden; Anett Grässler, Pirna; Elke Wilden,
Köln; Michael Hammer, St Josef-Stift Sendenhorst; Edmund Edelmann, Bad Aibling;
Christina Eisterhues, Braunschweig; Wolfgang Ochs, Bayreuth; Thomas Karger,
Eduardus-Krankenhaus Köln-Deutz; Michael Bäuerle, Universität Erlangen, Erlangen;
Herbert Kellner, München; Silke Zinke, Berlin; Angela Gause, Elmshorn; Lothar Meier,
Hofheim; Karl Alliger, Zwiesel; Martin Bohl-Bühler, Brandenburg; Carsten Stille,
Hannover; Susanna Späthling-Mestekemper and Thomas Dexel, München; Harald
Tremel, Hamburg; Stefan Schewe, Medizinische Poliklinik der Ludwig-Maximilians-
Universität München; Helmut Sörensen, Krankenhaus Waldfriede Berlin; Florian
Schuch, Erlangen; Klaus Krüger, München; Andreas Teipel, Leverkusen; Kirsten
Karberg, Berlin; Gisela Maerker-Alzer and Dorothea Pick, Holzweiler; Volker Petersen,
Hamburg; Kerstin Weiss, Lichtenstein; Werner Liman, Ev. Krankenhaus Hagen-Haspe; Hamburg; Kerstin Weiss, Lichtenstein; Werner Liman, Ev. Krankenhaus Hagen-Haspe;
Kurt Gräfenstein, Johanniter-Krankenhaus im Fläming, Treuenbrietzen; Jochen Walter,
Rendsburg; Werner A Biewer, Saarbrücken; Roland Haux, Berlin; Wolfgang Gross, Lübeck; Michael Zänker, Evangelisches Freikirchliches Krankenhaus Eberswalde; Gerhard Fliedner, Osnabrück; Thomas Grebe, Ev. Krankenhaus Kredenbach; Karin Leumann, Riesa; Jörg-Andres Rump, Freiburg; Joachim Gutfleisch, Biberbach; Michael Schwarz-Eywill, Evangelisches Krankenhaus Oldenburg; Kathrin Fischer, Greifswald; Monika Antons, Köln. The authors also acknowledge the significant contributions of Bernhard Manger, University of Erlangen, Jörn Kekow, University of Magdeburg and Peter Herzer, Munich for their service as members of the advisory board. Their work on the RABBIT advisory board is honorary and without financial compensation. The authors gratefully recognise the substantial contributions of Christina Bungartz, Ulrike Kamenz, Susanna Zernicke and all employees of the German Rheumatism Research Centre, Berlin in the study monitoring and support of the data analyses. The authors would like to thank Geert Molenberghs (University of Hasselt, Belgium) for sharing his experiences in using weighted GEE models and James Galloway (University of Manchester, UK) for his helpful comments related to this manuscript.

Funding RABBIT has been supported by an unconditional joint grant from Essex Pharma/MSD (since 2001), Wyeth Pharma/Pfizer (since 2001), Amgen (2003-8), Swedish Orphan Biovitrum (since 2009) and Abbott (since 9/2003). Since 2007 it has also been supported by Bristol-Myers Squibb and Roche. Since 2009 it has received support from UCB. The principal investigators and their team have full academic freedom in study design and conduct, data analysis and publication of results. These stipulations are delineated in their contract with the sponsors. However, for the purposes of information, all seven funding companies receive every manuscript 30 days prior to submission. The data interpretation, drafting, critical revision and approval of the final manuscript were performed solely by the authors.

Competing interests None.

Patient consent Obtained.

Ethics approval This study was approved in 2001 by the ethics committee of the Charité University Medicine, Berlin.

Provenance and peer review Not commissioned; externally peer reviewed.

\section{REFERENCES}

1. Bongartz T, Sutton AJ, Sweeting MJ, et al. Anti-TNF antibody therapy in rheumatoid arthritis and the risk of serious infections and malignancies: systematic review and meta-analysis of rare harmful effects in randomized controlled trials. JAMA 2006;295:2275-85.

2. Leombruno JP, Einarson TR, Keystone EC. The safety of anti-tumour necrosis factor treatments in rheumatoid arthritis: meta and exposure-adjusted pooled analyses of serious adverse events. Ann Rheum Dis 2009;68:1136-45.

3. Listing J, Strangfeld A, Kary S, et al. Infections in patients with rheumatoid arthritis treated with biologic agents. Arthritis Rheum 2005;52:3403-12.

4. Dixon WG, Watson K, Lunt M, et al. Rates of serious infection, including site-specific and bacterial intracellular infection, in rheumatoid arthritis patients receiving antitumor necrosis factor therapy: results from the British Society for Rheumatology Biologics Register. Arthritis Rheum 2006;54:2368-76.

5. Dixon WG, Symmons DP, Lunt M, et al. Serious infection following anti-tumor necrosis factor alpha therapy in patients with rheumatoid arthritis: lessons from interpreting data from observational studies. Arthritis Rheum 2007;56:2896-904.

6. Gómez-Reino JJ, Carmona L, Valverde VR, et al. Treatment of rheumatoid arthritis with tumor necrosis factor inhibitors may predispose to significant increase in tuberculosis risk: a multicenter active-surveillance report. Arthritis Rheum 2003;48:2122-7

7. Askling J, Fored CM, Brandt L, et al. Time-dependent increase in risk of hospitalisation with infection among Swedish RA patients treated with TNF antagonists. Ann Rheum Dis 2007;66:1339-44.

8. Curtis JR, Patkar N, Xie A, et al. Risk of serious bacterial infections among rheumatoid arthritis patients exposed to tumor necrosis factor alpha antagonists. Arthritis Rheum 2007;56:1125-33.

9. Greenberg JD, Reed G, Kremer JM, et al. Association of methotrexate and tumour necrosis factor antagonists with risk of infectious outcomes including opportunistic infections in the CORRONA registry. Ann Rheum Dis 2010;69:380-6.

10. Wolfe F, Caplan L, Michaud K. Treatment for rheumatoid arthritis and the risk of hospitalization for pneumonia: associations with prednisone, disease-modifying antirheumatic drugs, and anti-tumor necrosis factor therapy. Arthritis Rheum 2006;54:628-34.

11. Schneeweiss $\mathbf{S}$, Setoguchi S, Weinblatt ME, et al. Anti-tumor necrosis factor alpha therapy and the risk of serious bacterial infections in elderly patients with rheumatoid arthritis. Arthritis Rheum 2007;56:1754-64.

12. Zink A, Askling J, Dixon WG, et al. European biologicals registers: methodology, selected results and perspectives. Ann Rheum Dis 2009;68:1240-6.

13. Galloway JB, Hyrich KL, Mercer LK, et al. Anti-TNF therapy is associated with an increased risk of serious infections in patients with rheumatoid arthritis especially in the first 6 months of treatment: updated results from the British Society for Rheumatology Biologics Register with special emphasis on risks in the elderly. Rheumatology (Oxford) 2011;50:124-31. 
14. Prevoo ML, van 't Hof MA, Kuper HH, et al. Modified disease activity scores that include twenty-eight-joint counts. Development and validation in a prospective longitudinal study of patients with rheumatoid arthritis. Arthritis Rheum 1995;38:44-8.

15. Lautenschläger J, Mau W, Kohlmann T, et al. [Comparative evaluation of a German version of the Health Assessment Questionnaire and the Hannover Functional Capacity Questionnaire]. Z Rheumatol 1997;56:144-55.

16. Westhoff G, Listing J, Zink A. Loss of physical independence in rheumatoid arthritis: interview data from a representative sample of patients in rheumatologic care. Arthritis Care Res 2000;13:11-22.

17. Strangfeld A, Listing J, Herzer $P$, et al. Risk of herpes zoster in patients with rheumatoid arthritis treated with anti-TNF-alpha agents. JAMA 2009;301:737-44

18. Listing J, Strangfeld A, Rau R, et al. Clinical and functional remission: even though biologics are superior to conventional DMARDs overall success rates remain low-results from RABBIT, the German biologics register. Arthritis Res Ther 2006;8:R66

19. Robins JM, Hernán MA, Brumback B. Marginal structural models and causal inference in epidemiology. Epidemiology 2000;11:550-60.

20. Molenberghs G, Kenward MG. Missing Data in Clinical Studies. Chichester: John Wiley \& Sons, 2007.

21. Smitten AL, Choi HK, Hochberg MC, et al. The risk of hospitalized infection in patients with rheumatoid arthritis. J Rheumatol 2008;35:387-93.

22. Bernatsky S, Hudson M, Suissa S. Anti-rheumatic drug use and risk of serious infections in rheumatoid arthritis. Rheumatology (Oxford) 2007:46:1157-60.

23. Dixon WG, Hyrich KL, Watson KD, et al. Drug-specific risk of tuberculosis in patients with rheumatoid arthritis treated with anti-TNF therapy: results from the British Society for Rheumatology Biologics Register (BSRBR). Ann Rheum Dis 2010;69:522-8. 